\title{
A new complex Duffing oscillator used in complex signal detection
}

\author{
DENG XiaoYing ${ }^{*}$, LIU HaiBo \& LONG Teng \\ School of Information and Electronics, Beijing Institute of Technology, Beijing 100081, China
}

Received December 12, 2011; accepted February 2, 2012

\begin{abstract}
In view of the fact that complex signals are often used in the digital processing of certain systems such as digital communication and radar systems, a new complex Duffing equation is proposed. In addition, the dynamical behaviors are analyzed. By calculating the maximal Lyapunov exponent and power spectrum, we prove that the proposed complex differential equation has a chaotic solution or a large-scale periodic one depending on different parameters. Based on the proposed equation, we present a complex chaotic oscillator detection system of the Duffing type. Such a dynamic system is sensitive to the initial conditions and highly immune to complex white Gaussian noise, so it can be used to detect a weak complex signal against a background of strong noise. Results of the Monte-Carlo simulation show that the proposed detection system can effectively detect complex single frequency signals and linear frequency modulation signals with a guaranteed low false alarm rate.
\end{abstract}

complex Duffing equation, chaos detection, weak complex signal, signal-to-noise ratio, probability of detection

Citation: Deng X Y, Liu H B, Long T. A new complex Duffing oscillator used in complex signal detection. Chin Sci Bull, 2012, 57: 2185-2191, doi: 10.1007/s11434-012-5145-8

Chaos theory is one of the most significant achievements of nonlinear science. Many scholars have researched the basic theoretical issues [1-7] and practical applications of chaos. Hitherto, chaos theory has been widely applied in the control [8], synchronization [9], prediction [10], communication $[11,12]$, and detection fields, among others. In signal detection, detection of weak signals using a chaotic oscillator in the real domain [13-19] has been studied by some researchers, with most researchers using a Duffing oscillator or modified version thereof. The basic idea is that a chaotic system is sensitive to the initial conditions and less influenced by noise. Nowadays complex signal processing is being used in many fields of science and engineering including digital communication systems, radar systems, antenna beamforming applications, coherent pulse measurement systems, and so on. As such, it is very meaningful to study how to detect a complex signal against a noisy background.

Most of the recent works on complex chaos have focused on solving complex nonlinear differential equations, com-

*Corresponding author (email: xydeng@bit.edu.cn) plex chaos control and synchronization, and so on. For example, Cveticanin [20] developed an approximate analytic approach for solving strong nonlinear differential equations of the Duffing type with a complex-valued function. Furthermore, excellent agreement is found between the analytic results and numerical ones. Li et al. [21] developed a stochastic averaging method for a quasi-Hamilton system to study the stationary solution in a nonlinear stochastically complex dynamical system. Rauh et al. [22] proved by means of a quadratic Lyapunov function that the complex Lorenz model is globally stable and presented an analytic expression for the upper bound on the magnitude of the time-dependent electric field. Mahmoud and Aly [23] illustrated the existence of periodic attractors of complex damped nonlinear systems by constructing Poincaré plots, and investigated the stability properties of the solutions of a complex nonlinear equation. The strange attractors, chaotic behavior and the problem of chaos control for a complex Duffing oscillator are studied in [24]. Ref. [25] investigated the phenomenon of chaos synchronization of two different chaotic complex systems of the Chen and Lü type via methods for active control and global synchronization. 
Based on the work in [24], Li et al. [26] studied the problem of chaos control for a complex Duffing oscillation system. Up to now, no study on detecting complex signals using a complex chaotic system has been published. First, we propose a non-symmetrical complex Duffing equation based on the real Duffing equation. By calculating the maximal Lyapunov exponent (MLE) and power spectrum, we prove that the proposed complex differential equation has a chaotic solution or a large-scale periodic one depending on different parameters. Furthermore, it is very sensitive to different initial conditions. Then, based on the proposed complex Duffing equation, a complex Duffing oscillator detection model is constructed. Last, under the condition of a guaranteed low false alarm rate, we investigate the performance of detection of such a dynamic system for noisy complex single frequency signals and complex linear frequency modulation (LFM) signals using Monte-Carlo simulations.

\section{Theoretical basis}

\subsection{Complex Duffing equation}

The most common form of the real Duffing equation [14] is:

$$
\ddot{x}+k \dot{x}-x+x^{3}=\gamma \cos (t),
$$

where $k$ is the viscous damping factor, $\left(-x+x^{3}\right)$ plays the role of nonlinear elastic restitution, and $\gamma \cos (t)$ is the internal periodic force. According to this equation, a new complex Duffing equation is constructed as

$$
\ddot{z}+k \dot{z}-z+\varepsilon z|z|^{2}=\gamma \exp (j t),
$$

where $z=x+j y(j=\sqrt{-1})$ is a complex function, $k, \varepsilon$, and $\gamma(\gamma \geqslant 0)$ are real parameters, and the dots are time derivatives. For the purpose of adapting to weak signals with different frequencies instead of a certain signal with angular frequency $\omega=1 \mathrm{rad} / \mathrm{s}$, let $t=\omega \tau$ ( $\omega$ is an arbitrary value). Eq. (2) then becomes

$$
\frac{1}{\omega^{2}} \ddot{z}+\frac{k}{\omega} \dot{z}-z+\varepsilon z|z|^{2}=\gamma \exp (j \omega \tau) .
$$

Eq. (3) represents a system of two coupled nonlinear second-order differential equations (for the sake of readability, $t$ denotes $\tau$ ):

$$
\begin{aligned}
& \frac{1}{\omega^{2}} \ddot{x}+\frac{k}{\omega} \dot{x}-x+\varepsilon x\left(x^{2}+y^{2}\right)=\gamma \cos (\omega t), \\
& \frac{1}{\omega^{2}} \ddot{y}+\frac{k}{\omega} \dot{y}-y+\varepsilon y\left(x^{2}+y^{2}\right)=\gamma \sin (\omega t) .
\end{aligned}
$$

Then, we study the nonlinear dynamical behavior of the new complex Duffing equation. By introducing four new variables, $x_{1}=x, x_{2}=\frac{\dot{x}}{\omega}, x_{3}=y, x_{4}=\frac{\dot{y}}{\omega}$, eq. (4a) and (4b) become

$$
\left\{\begin{array}{l}
\dot{x}_{1}=\omega x_{2}, \\
\dot{x}_{2}=\omega\left[x_{1}-k x_{2}-\varepsilon x_{1}\left(x_{1}^{2}+x_{3}^{2}\right)+\gamma \cos (\omega t)\right], \\
\dot{x}_{3}=\omega x_{4}, \\
\dot{x}_{4}=\omega\left[x_{3}-k x_{4}-\varepsilon x_{3}\left(x_{1}^{2}+x_{3}^{2}\right)+\gamma \sin (\omega t)\right] .
\end{array}\right.
$$

(i) Case of $\gamma=0$. For the case of $\gamma=0$, the equations in eq. (5) are completely symmetrical; that is, if one starts from initial conditions $\left(x_{0}, \dot{x}_{0}\right)=\left(y_{0}, \dot{y}_{0}\right)$, the $x$ and $y$ variables behave identically. From eq. (5) $(\gamma=0)$, we can easily obtain a saddle point of the phase plane at $(x, \dot{x}, y, \dot{y})=(0,0,0,0)$, and two focal points at $\left(\sqrt{\frac{1}{2 \varepsilon}}, 0, \sqrt{\frac{1}{2 \varepsilon}}, 0\right)$ and $\left(-\sqrt{\frac{1}{2 \varepsilon}}, 0,-\sqrt{\frac{1}{2 \varepsilon}}, 0\right)$, respectively.

Points $(x, \dot{x})$ and $(y, \dot{y})$ finally remain at one of the two focal points depending on the initial values. Using a fourth-order Runge-Kutta algorithm to solve eq. (5), we can obtain the solutions for different initial conditions as shown in Figure 1(a) and (b), where $\omega=1 \mathrm{rad} / \mathrm{s}, k=0.5, \varepsilon=1$, the step length $h=0.05 \mathrm{~s}$, and the simulation time $t_{s}=200 \mathrm{~s}$. In the following experiments, the same parameters as given above are used unless specified otherwise. From Figure 1, we can see that for initial values $[1,1,1,1]$, the points $(x, \dot{x})$ finally remain at the left focus $(-0.707,0)$, whereas for initial values $[-1,-1,-1,-1]$, the points $(x, \dot{x})$ finally remain at the right focus $(0.707,0)$. We plot only the phase plane trajectory of $(x, \dot{x})$ for the different initial conditions since the equations in eq. (5) are symmetrical, and the same trajectory for $(y, \dot{y})$ can be obtained.

(ii) Case of $\gamma>0$. For the case of $\gamma>0$, eq. (5) show very complicated dynamical behavior. Figure 2 shows the bifurcation diagram of variations in variable $x$ with changes in parameter $\gamma$. It can be seen that the complex Duffing oscillator eq. (3) has both chaotic behavior and large-scale periodic behavior, and that it remains in a large-scale periodic state for a wide range of parameter $\gamma$. From the zoom-in plot within $\gamma=[0.7,0.8]$, we can obtain the critical value $\gamma_{c} \in(0.72,0.73)$ at which the system is in a critical state (chaotic, but on the verge of changing to a large-scale periodic state).

\subsection{Chaotic state and large-scale periodic state}

In this section, we give examples of chaotic and large-scale periodic solutions for eq. (3). Let $k=0.5, \varepsilon=1,\left(x_{0}, \dot{x}_{0}, y_{0}\right.$, $\left.\dot{y}_{0}\right)=(0,0,0,0)$, and $\omega=1 \mathrm{rad} / \mathrm{s}$. For $\gamma=0.6$, eq. (3) has chaotic behavior with the phase plane trajectories of $(x, \dot{x})$ and $(y, \dot{y})$ as shown in Figure 3(a) and (b), respectively. 

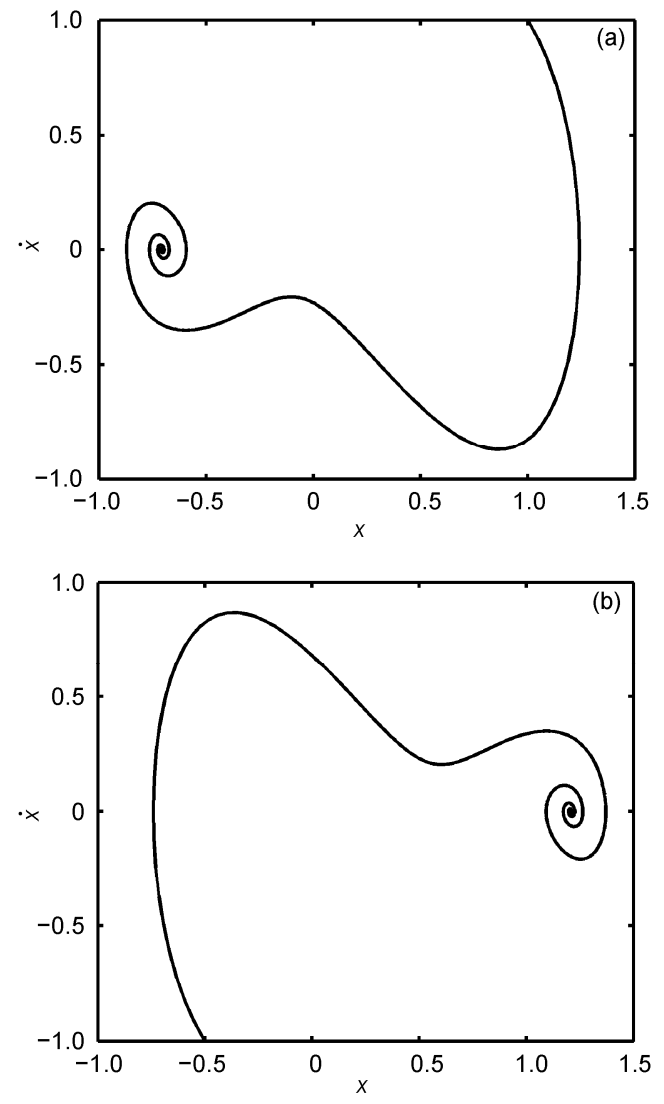

Figure 1 Phase plane trajectories of $(x, \dot{x})$ for different initial conditions $(\gamma=0)$. (a) Trajectory for initial values $\left(x_{0}, \dot{x}_{0}, y_{0}, \dot{y}_{0}\right)=(1,1,1,1)$; (b) trajectory for initial values $\left(x_{0}, \dot{x}_{0}, y_{0}, \dot{y}_{0}\right)=(-1,-1,-1,-1)$.

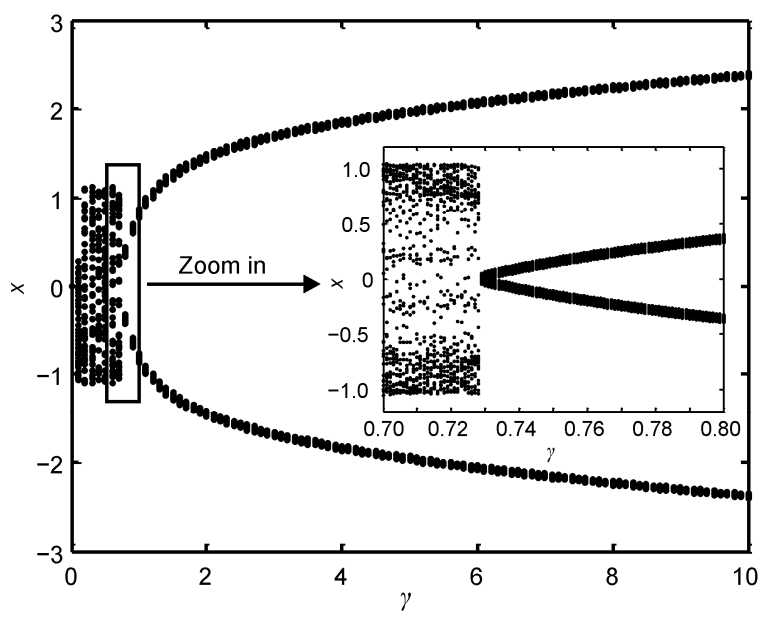

Figure 2 Bifurcation diagram for the complex Duffing eq. (3).

There are a few differences between the two trajectories. Any system, containing at least one positive MLE or containing a power spectrum with prominent peaks separated by broad-band domains, is defined to be chaotic [24]. From Figure 3(c), the MLEs are always positive within the simulation time, and from Figure 3(d), the power spectrum $P_{x}(\omega)$ of $x$ has three prominent peaks separated by broad-band domains, indicating that the system is in a chaotic phase state.

For the same conditions as used above, with $\gamma=0.73$, eq. (3) has large-scale periodic behavior. The diagrams corresponding to Figure 3 are shown in Figure 4. From Figure 4(a) and (b), the phase trajectories quickly stabilize on periodic orbits with a certain width after undergoing a short transient state. From Figure 4(c), the MLEs remain negative after $t>97 \mathrm{~s}$, and from Figure 4(d), the power spectrum $P_{x}(\omega)$ of $x$ has only one peak at $\omega=1 \mathrm{rad} / \mathrm{s}$, indicating that the system is in a large-scale phase state.

\section{Detection model for the complex Duffing oscillator and simulation}

From section 1, the complex Duffing eq. (3) is sensitive to parameter $\gamma$, and a tiny periodic perturbation can change the dynamical behavior. So one can detect whether the desired signal really exists by checking the state of the system in the phase space. The following detection model for the complex Duffing oscillator is constructed:

$$
\frac{1}{\omega^{2}} \ddot{z}+\frac{0.5}{\omega} \dot{z}-z+z|z|^{2}=\gamma_{c} \exp (j \omega t)+c s(t),
$$

where $\gamma_{c}$ is the critical value and $\operatorname{cs}(t)$ is the input complex signal to be detected. Before the signal to be detected is input, the system is put into the critical state. Note that because of the truncation error involved in the Runge-Kutta algorithm, different initial conditions may cause a distinct discrepancy in the critical value [15]. The accuracy of $\gamma_{c}$ may be chosen in accordance with practical demand, and we set $\gamma_{c}=0.72$ in our experiments. Next we investigate the detection performance of the model (6) using Monte-Carlo simulations.

(i) Experiment 1. Complex white Gaussian noise is used as the input signal to be detected. First, we only input noise into the detection system, that is $\operatorname{cs}(t)=n(t)$, so that the effect of complex white Gaussian noise on the complex detection system can be investigated. Noise has a nondeterministic effect on a nonlinear system, and it may induce different dynamical behavior such as chaos, order, or escape from the attractor [27]. The purpose of this paper is to detect the desired signal in noise, so we need the complex detection system to be immune to noise. In other words, the system should remain in a chaotic state after the noise is input. Thus, we do not want the noise to induce the phase transition. If so, misjudgment occurs, which is known as a false alarm in a radar system. A false alarm occurs when a non-target event exceeds the detection criterion and is mistakenly identified as a target. We use this term to denote the phenomenon in which noise induces the phase transition.

In many applications, for a given detection probability, a 

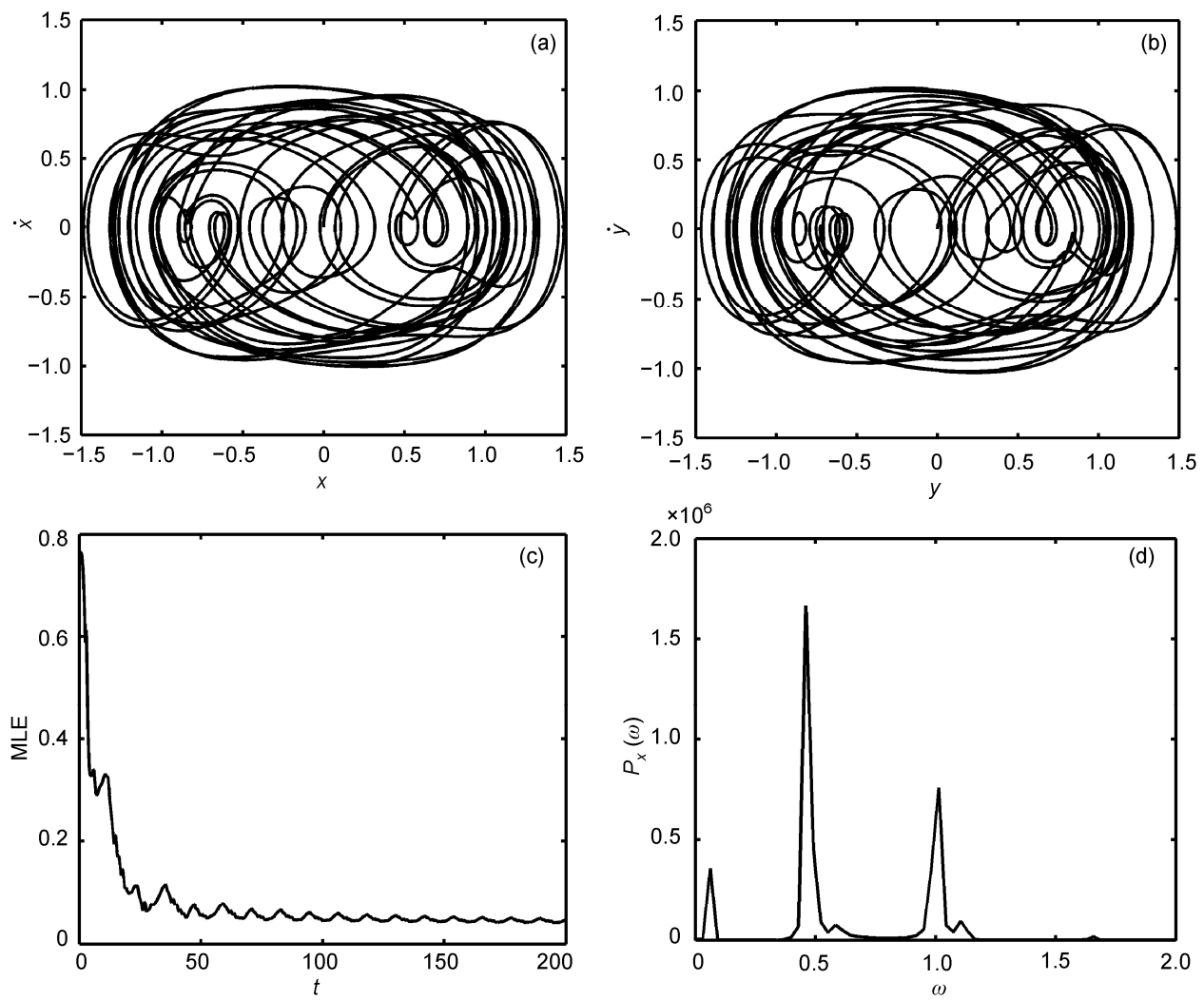

Figure 3 Example of a chaotic state $(k=0.5, \varepsilon=1, \omega=1 \mathrm{rad} / \mathrm{s}, \gamma=0.6)$. (a) Phase plane trajectory of $(x, \dot{x})$; (b) phase plane trajectory of $(y, \dot{y}) ;(\mathrm{c})$ MLEs; (d) power spectrum of $x$.
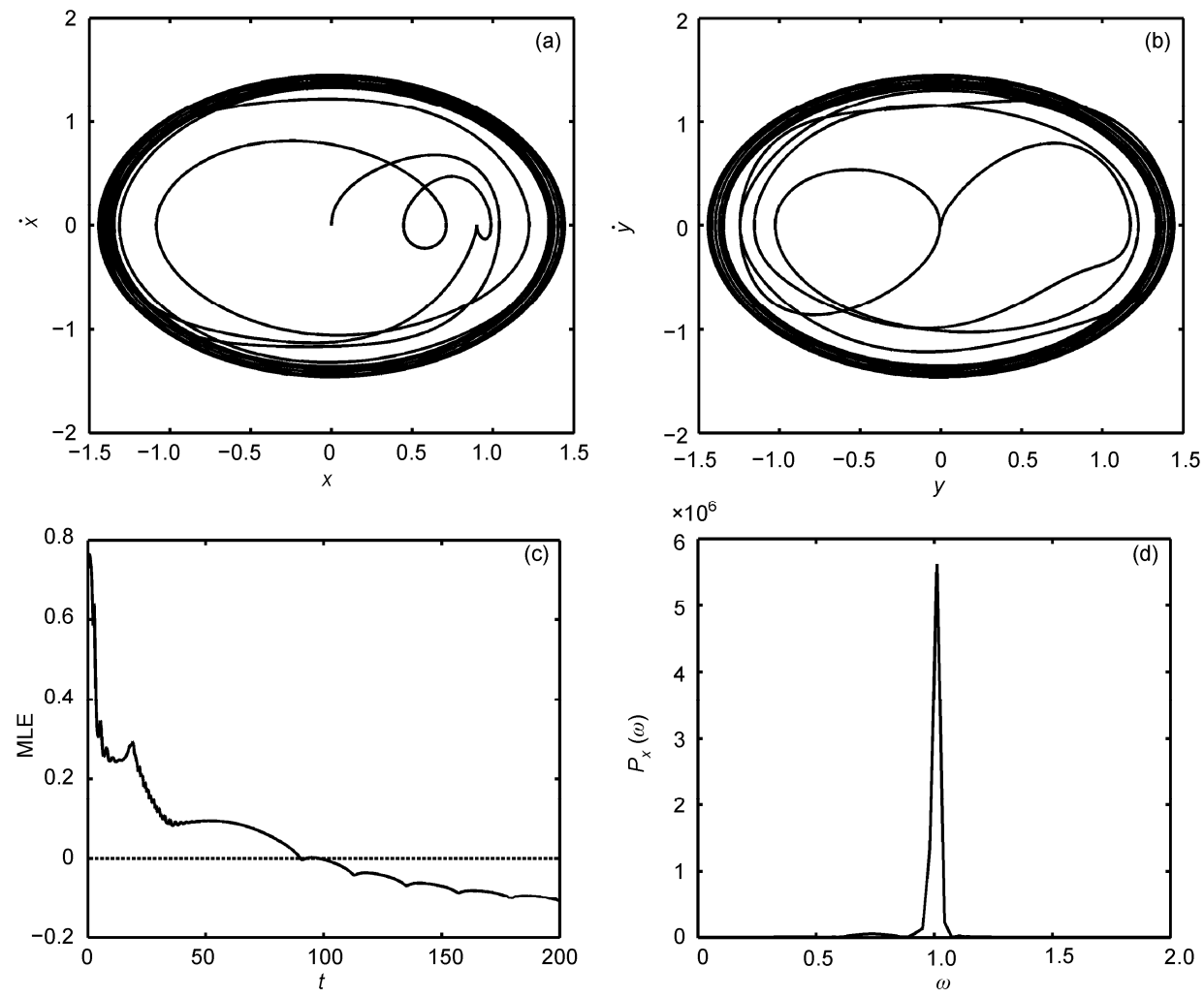

Figure 4 Example of a large-scale periodic state $(k=0.5, \varepsilon=1, \omega=1 \mathrm{rad} / \mathrm{s}, \gamma=0.73)$. (a) Phase plane trajectory of $(x, \dot{x})$; (b) phase plane trajectory of $(y, \dot{y}) ;(\mathrm{c})$ MLEs; (d) power spectrum of $x$. 
lower false alarm rate is better. Conversely, for a given tolerable false alarm rate, a higher detection probability is better. By means of Monte-Carlo simulations, we investigated the false alarm rates of the system (6) when noise is input at different levels. Table 1 lists the false alarm rates of the detection system under different conditions. Note that for the case of noise power $\sigma^{2}=0.005 \mathrm{~W}$, we carried out 10000 simulations with only a false alarm occurring, and the false alarm rate equal to $10^{-4}$. So we can roughly conclude that the detection system is strongly immune to complex white Gaussian noise below $0.005 \mathrm{~W}$, which lays a good foundation for the reliable detection of the desired signal in noise.

In practical detection, the noise power should first be estimated using data in which no desired signal exists. Then, if the estimation is greater than $0.005 \mathrm{~W}$, the noisy signal to be detected needs to be multiplied by an attenuation factor to ensure that the noise power is below $0.005 \mathrm{~W}$, so that a low false alarm rate can be guaranteed. Note that the value of noise power $0.005 \mathrm{~W}$ corresponds to $\gamma_{c}=0.72$, and if the accuracy of $\gamma_{c}$ is improved (by expressing the value to more decimal places), we can ascertain through simulation that the tolerable noise power will decline to retain the same false alarm rate.

(ii) Experiment 2. Detection of complex single frequency signals in complex white Gaussian noise. Let the input of the complex detection system be $c s(t)=A \exp (j \omega t)+n(t)$, where the complex single frequency signal $A \exp (j \omega t)$ has the same frequency and initial phase as the internal periodic force, $A$ is the amplitude of the signal, and $n(t)$ is complex white Gaussian noise. Then, the total force is $\left(\gamma_{c}+A\right) \exp (j \omega t)+n(t)$. We fixed the noise power $\sigma^{2}=$ $0.005 \mathrm{~W}$ and gradually changed the amplitude $A$ of the complex signal to be detected, to investigate the detection probabilities of the system under different signal-to-noise ratios (SNR). The SNR is defined as:

$$
\mathrm{SNR}=10 \log _{10}\left(\frac{A^{2}}{2 \sigma^{2}}\right) \mathrm{dB} .
$$

The number of Monte-Carlo simulations used to estimate each detection probability was 200 . In every simulation, the noise was first input into the system to test whether a false

Table 1 False alarm rate induced by complex white Gaussian noise at different levels

\begin{tabular}{ccccc}
\hline No. & $\begin{array}{c}\text { Noise power } \\
(\mathrm{W})\end{array}$ & $\begin{array}{c}\text { Number of } \\
\text { simulations }\end{array}$ & $\begin{array}{c}\text { Number of false } \\
\text { alarms }\end{array}$ & $\begin{array}{c}\text { False alarm } \\
\text { rate }(\%)\end{array}$ \\
\hline 1 & 0.1 & 1000 & 452 & 45.2 \\
2 & 0.05 & 1000 & 301 & 30.1 \\
3 & 0.01 & 1000 & 26 & 2.6 \\
4 & 0.005 & 10000 & 1 & 0.01 \\
5 & 0.001 & 1000 & 0 & 0 \\
\hline
\end{tabular}

alarm occurred, and then the signal corrupted by this noise was input into the system to test whether the desired signal was detected. Figure 5 plots the curve of the obtained detection probabilities with different SNRs. No false alarms occurred in our experiments. From Figure 5, the lower the SNR, the lower the detection probability. Furthermore, to achieve a detection probability over $90 \%$, the SNR should usually be greater than $-20 \mathrm{~dB}$.

(iii) Experiment 3. Detection of complex LFM signals in complex white Gaussian noise. Let the input of the complex detection system be $c s(t)=A \exp \left[j\left(\omega_{0} t+0.5 b t^{2}\right)\right]$ $+n(t)$, where $\omega_{0}$ is the initial frequency of the LFM signal and $b$ is the slope. Generally, the approach for chaotic detection has an obvious advantage in detecting signals with the same frequency as the internal periodic force. But the frequency of the LFM signal changes with time, so the detection performance for the LFM signal should be worse than that for the single frequency signal. This can easily be investigated through experiments.

Next, we investigated the effect of different slopes and frequency ranges on the detection performance of the system. To obtain results accurately, the simulation time $t_{s}$ was chosen as $400 \mathrm{~s}$, and the signals to be detected once again had a length of $200 \mathrm{~s}$ (padded with trailing zeros to length $400 \mathrm{~s})$. With the frequency of the internal periodic force $\omega=1 \mathrm{rad} / \mathrm{s}$, noise power $\sigma^{2}=0.005 \mathrm{~W}$, and $\omega_{0}=0 \mathrm{rad} / \mathrm{s}$, we altered the value of slope $b$. The number of Monte-Carlo simulations used to estimate each detection probability was 200. The process for the simulation was the same as in experiment 2. Once again, no false alarms occurred in these simulations. Table 2 lists the detection probabilities for different slopes values. From Table 2, for the case of SNR= $-10 \mathrm{~dB}$, the detection probability for slope $b=0.01 \mathrm{rad} / \mathrm{s}^{2}$ is the highest and no signal is detected for the slope $b=0.05$ and $0.1 \mathrm{rad} / \mathrm{s}^{2}$. For the case of $\mathrm{SNR}=2 \mathrm{~dB}$, the detection probabilities increase because of the improved SNR, even reaching $100 \%$ for the slope $b=0.005$ and $0.01 \mathrm{rad} / \mathrm{s}^{2}$.

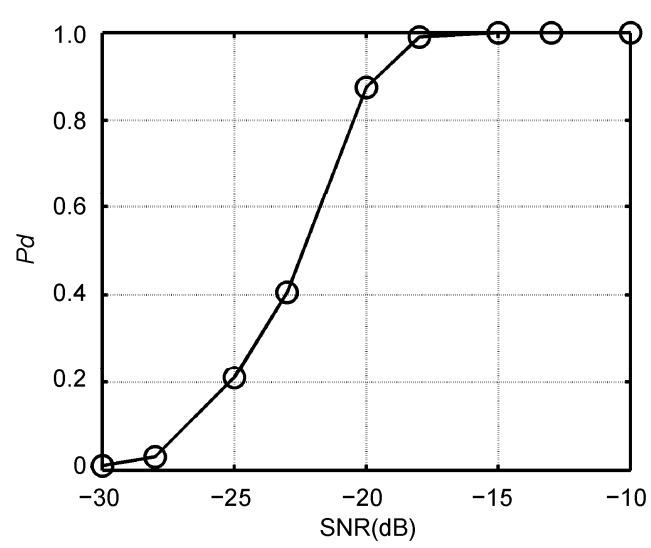

Figure 5 Curve of detection probabilities for complex single frequency signals. 
Table 2 Detection probabilities of the complex chaotic detection system for different complex LFM signals

\begin{tabular}{|c|c|c|c|c|}
\hline \multirow{2}{*}{ Number } & \multirow{2}{*}{$\begin{array}{c}\text { Slope } \\
\left(\mathrm{rad} / \mathrm{s}^{2}\right)\end{array}$} & \multirow{2}{*}{$\begin{array}{c}\text { Frequency } \\
\text { range (rad/s) }\end{array}$} & \multicolumn{2}{|c|}{ Detection probability } \\
\hline & & & $\mathrm{SNR}=-10 \mathrm{~dB}$ & $\mathrm{SNR}=2 \mathrm{~dB}$ \\
\hline 1 & 0.005 & {$[0,1]$} & $85.5 \%$ & $100 \%$ \\
\hline 2 & 0.01 & {$[0,2]$} & $89.5 \%$ & $100 \%$ \\
\hline 3 & 0.025 & {$[0,5]$} & $71 \%$ & $90 \%$ \\
\hline 4 & 0.05 & {$[0,10]$} & 0 & $4 \%$ \\
\hline 5 & 0.1 & {$[0,20]$} & 0 & 0 \\
\hline
\end{tabular}

\section{Discussion and conclusions}

(i) Comparison of two complex Duffing equations. In 2001, Mahmoud et al. [24] presented the following complex Duffing equation

$$
\ddot{z}-\alpha z+\varepsilon z|z|^{2}=\gamma \exp (j \omega t)
$$

where $z=x+j y$, and $\varepsilon, \gamma, \alpha$, and $\omega$ are positive real parameters. Let $\varepsilon=1, \alpha=1$, and $\omega=1 \mathrm{rad} / \mathrm{s}$. Then we can obtain the critical value of eq. (8) $\gamma_{c} \in(0.22,0.23)$ from its bifurcation diagram. Next we compare the required time for eqs. (3) and (8) to enter large-scale periodic solutions after undergoing a transient state. For eq. (3), for the case of $\gamma=0.73$, the solution space begins to enter a large-scale periodic state at simulation time $t_{s} \approx 97 \mathrm{~s}$. With an increase in $\gamma$, the required time decreases quickly. For example, the time is $t_{s} \approx 31 \mathrm{~s}$ for $\gamma=0.8$. Comparatively, for eq. (8), the required time is $t_{s} \approx 229 \mathrm{~s}$ for $\gamma=0.23$. With an increase in $\gamma$, the time decreases slowly. For example, the time is $t_{s} \approx 191 \mathrm{~s}$ for $\gamma=0.8$. It is well known that the speed with which a chaotic equation transforms into a stable large-scale periodic solution is very important for detecting signals quickly and accurately. In this sense, the proposed eq. (3) in this paper is more suited to signal detection than eq. (8). For eq. (8), the reason for having a longer transient state may be the lack of a viscous damping term $k \dot{z}$.

(ii) On the detection capability of the complex Duffing system for complex LFM signals. In general, the parts of LFM signals with nearly the same frequency as the internal periodic force in a chaotic system have an effect on the phase state transition. In addition, the amplitude of these signals must be large enough, and the duration of these signals must be long enough. Otherwise, if the amplitude of signals is too small to induce the phase transition, or if the duration of signals is too short to complete the phase transition, the system will remain in the chaotic state. In experiment 3 of section 2, without consideration of noise, the instantaneous frequency of the LFM signal is $\omega(t)=$ $\frac{\mathrm{d}\left(\omega_{0} t+0.5 b t^{2}\right)}{\mathrm{d} t}=\omega_{0}+b t$, so the instantaneous frequency is dependent on the slope $b$. The larger the slope, the faster the variation in frequency of signals. Under the condition of a fixed SNR ( such as SNR= $-10 \mathrm{~dB}$ ), the duration of signals with nearly the same frequency as the internal periodic force $(1 \mathrm{rad} / \mathrm{s})$ will become shorter with an increase in the slope. A larger slope will result in lower detection probability. Though the slope of case 1 in Table 2 is smaller than that of case 2 , the lack of signals with frequencies greater than $1 \mathrm{rad} / \mathrm{s}$ results in a lower detection probability than case 2. For cases 4 and 5, the frequency of the LFM signals changes quickly from 0 to $10 \mathrm{rad} / \mathrm{s}$ and $20 \mathrm{rad} / \mathrm{s}$, respectively. The duration of signals with frequency around $1 \mathrm{rad} / \mathrm{s}$ is too short to induce the phase transition, so the signal can hardly be detected. On the other hand, under the condition of a fixed slope, the higher the SNR is, the greater is the obtained detection probability. For example, for the slope $b=0.005 \mathrm{rad} / \mathrm{s}^{2}$, the detection probability improves from $85.5 \%$ to almost $100 \%$ when the SNR is enhanced from -10 to $2 \mathrm{~dB}$. The problem of how to improve the performance of detection of such a wideband signal needs to be further investigated.

In this paper, a new complex Duffing equation was proposed, and its dynamical behavior was analyzed. Based on the proposed equation, we constructed a complex chaotic oscillator detection system to detect complex signals in noise. We investigated the influence of noise on the detection system and the detection performance of the system for complex signals. The experimental results show that the complex chaotic oscillator is highly immune to noise at a certain level, and has good detection performance for weak complex single frequency signals and LFM signals. This work lays the foundation for complex digital signal processing in digital communication systems, radar systems, and so on.

This work was supported by the National Natural Science Foundation of China (40804022, 61032009 and 61001198). We sincerely thank to anonymous reviewers for their constructive suggestions and careful revisions that greatly improve the readability of this article.

1 Lorenz E N. Deterministic nonperiodic flow. J Atmos Sci, 1963, 20: 130-141

2 Li T Y, Yorke J A. Period three implies chaos. Amer Math Mon, 1975, 82: 985-992

3 Sun G Z, Liu Z R. Chaotic state of soft spring Duffing system. Chin Sci Bull, 1987, 32: 1464-1469

4 Hilborn R C, Ding M Z. Optimal reconstruction space for estimating correlation dimension. Int J Bifurcat Chaos, 1996, 6: 377-381

5 Gottlieb H P W, Sprott J C. Simplest driven conservative chaotic oscillator. Phys Lett A, 2001, 291: 385-388

6 Ji C C, Zhu H, Jiang W. A novel method to identify the scaling region for chaotic time series correlation dimension calculation. Chin Sci Bull, 2011, 56: 925-932

7 Shi L, Wang R W, Zhu L X, et al. Varying coefficient analysis for indeterminate species interactions with non-parametric estimation, exemplifying with a fig-fig wasp system. Chin Sci Bull, 2011, 56: 2545-2552

8 Shinbrot T, Grebogi C, Yorke $\mathrm{J}$ A, et al. Using small perturbations to 
control chaos. Nature, 1993, 363: 411-417

9 Ricardo F, José A R, Guillermo F A. Adaptive synchronization of high-order chaotic systems: A feedback with low-order parameterization. Physica D, 2000, 139: 231-246

10 Thiffeault J L. Chaos in the gulf. Science, 2010, 330: 458-459

11 Yan S L. Enhancement of chaotic carrier bandwidth in a semiconductor laser transmitter using self-phase modulation in an optical fiber external round cavity. Chin Sci Bull, 2010, 55: 1007-1012

12 Lei L H, Shi H L, Ma G Y. CAPS satellite spread spectrum communication blind multi-user detecting system based on chaotic sequences. Sci China-Phys Mech Astron, 2009, 52: 339-345

13 Brown R, Chua L O, Popp B. Is sensitive dependence on initial conditions nature's sensory device? Int J Bifurcat Chaos, 1992, 2: 193-199

14 Wang G Y, Tao G L, Chen X, et al. The application of chaotic oscillator to weak signal detection under the strong noise background (in Chinese). Chin J Sci Instrum, 1997, 18: 209-212

15 Wang G Y, He S L. A quantitative study on detection and estimation of weak signals by using chaotic Duffing oscillators. IEEE Trans Circuit Syst-I, 2003, 50: 945-953

16 Li Y, Yang B J. Chaotic system for the detection of periodic signals under the background of strong noise. Chin Sci Bull, 2003, 48: 508510

17 Li Y, Yang B J, Yuan Y, et al. Ability to detect weak effective seismic signals by utilizing chaotic vibrator system. Chin Sci Bull, 2006, 51: 3010-3017

18 Zheng S Y, Guo H X, Li Y A, et al. A new method for detecting line spectrum of ship-radiated noise using Duffing oscillator. Chin Sci Bull, 2007, 52: 1906-1912

19 Li Y, Yang B J, Badal J, et al. Chaotic system detection of weak seismic signals. Geophys J Int, 2009, 178: 1493-1522

20 Cveticanin L. Analytic approach for the solution of the complexvalued strong nonlinear differential equation of Duffing type. Physica A, 2001, 297: 348-360

$21 \mathrm{Li} \mathrm{W}, \mathrm{Xu} \mathrm{W}$, Zhao J F, et al. The study on stationary solution of a stochastically complex dynamical system. Physica A, 2007, 385: 465-472

22 Rauh A, Hannibal L, Abraham N B. Global stability properties of the complex Lorenz model. Physica D, 1996, 99: 45-58

23 Mahmoud G M, Aly S A. Periodic attractors of complex damped nonlinear systems. Int J Nonlinear Mech, 2000, 35: 309-323

24 Mahmoud G M, Mohamed A A, Aly S A. Strange attractors and chaos control in periodically forced complex Duffing's oscillators. Physica A, 2001, 292: 193-206

25 Mahmoud G M, Bountis T, AbdEi-Latif G M, et al. Chaos synchronization of two different chaotic complex Chen and Lü systems. Nonlinear Dynamics, 2009, 55: 43-53

26 Li X M, Wang C, Gong J. Chaotic behavior and chaos control in periodically forced complex Duffing's oscillation systems (in Chinese). J Xi' an Jiaotong Univ, 2003, 37: 264-267

27 Xie T, Wei X Y. Study on reliability of chaotic oscillator in weak signal detection (in Chinese). Chin J Sci Instrum, 2008, 29: 12651269

Open Access This article is distributed under the terms of the Creative Commons Attribution License which permits any use, distribution, and reproduction in any medium, provided the original author(s) and source are credited. 